\title{
Valproate-Induced Non-Hepatic Hyperammonaemic Encephalopathy: A Rare Complication of Chronic Valproate Therapy
}

\author{
Amit Agrawal $^{\mathrm{a}, \mathrm{c}}$, Rashmi Agrawal ${ }^{\mathrm{b}}$
}

\begin{abstract}
Valproic acid can sometimes produce encephalopathy secondary to hyperammonemia without hepatic dysfunction, termed as valproate-induced hyperammonaemic encephalopathy (VHE). VHE is characterized by acute onset of impaired consciousness, lethargy, confusion, headache, vomiting, seizures and focal neurological deficits. Known risk factors include therapy with multiple antiepileptic drugs, presence of underlying inborn errors of metabolism, febrile states, and insufficient nutritional intake. We hereby, describe a case of VHE in a 10-year-old male child on long-term VPA therapy and no history of underlying liver disease. Encephalopathy reversed on withdrawal of VPA and L-carnitine therapy. We emphasize the need for early recognition, investigation, and treatment of this potentially life-threatening condition.
\end{abstract}

Keywords: Encephalopathy; Hyperammonemia; Valproic acid

\section{Introduction}

Valproic acid (VPA) is an anti-epileptic drug (AED) commonly used in children to treat generalized and partial epilepsy syndromes because of its broad spectrum of activity [1]. Although, valproate-induced hepatic encephalopathy is a wellrecognized entity, it can sometimes produce encephalopathy without hepatic dysfunction, termed as valproate-induced hyperammonemic encephalopathy (VHE). VHE is charac-

Manuscript accepted for publication June 11, 2012

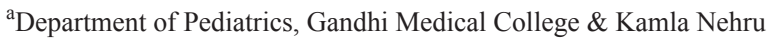
Hospital, Bhopal, India

${ }^{b}$ Devmata Hospital, Bhopal, Devmata Hospital Ahinsa Vihar Colony, Ayodhya Bypass Road, Bhopal - 462030, MP, India

${ }^{\mathrm{c} C}$ Corresponding author: Amit Agrawal, 28, Ravidas Nagar, Near

Nizamuddin Colony, Indrapuri, Bhopal, MP, India - 462021.

Email: agrawaldramit@yahoo.co.in

doi:10.4021/ijcp33w terized by acute onset of impaired consciousness, headache, vomiting, seizures, and focal neurological deficits or ataxia, with rapid recovery after discontinuation of VPA. Although, pathogenesis of VHE is not well understood, hyperammonemia has been implicated in causation of encephalopathy [2].

Most of the cases reported in literature developed encephalopathy early after initiation of VPA. However, our case illustrates that VHE can occur during chronic VPA therapy also. Therefore, in any patient on VPA therapy presenting with signs of encephalopathy, ammonia level should be checked and VPA should be discontinued. As in our case, encephalopathy reversed following withdrawal of VPA, which emphasizes the importance of early diagnosis and treatment.

\section{Case Report}

A 10-year-old male child brought with recurrent vomiting, headache, confusion and one episode of generalised convulsions followed by drowsiness. He was born at term with normal birth history and normal developmental milestones. He was a known case of idiopathic generalized epilepsy since 7 years of age. He was treated initially with phenytoin and switched over to sodium valproate (200 mg twice a day) since last one year. On examination, child was drowsy with normal vitals. His fundus examination was normal and pupils were symmetrical, normally reacting to light. There was no focal deficit, and no meningeal signs were there. The motor and sensory examination including deep tendon reflexes were normal.

Investigations including hemogram, liver and renal function tests, serum electrolytes and blood sugar were within normal limits. Peripheral smear did not reveal any hemoparasites or malignant cells. CSF analysis showed normal results. MRI brain was performed but did not reveal any abnormality. An EEG showed diffuse slowing of background activity consistent with encephalopathy. Subsequently, serum ammonia level was estimated which was raised to 114 $\mu \mathrm{mol} / \mathrm{L}$ (normal range $12-47 \mu \mathrm{mol} / \mathrm{L}$ ). The levels of serum valproate were found to be within normal range $(63 \mu \mathrm{g} / \mathrm{mL}$, normal range $50-100 \mu \mathrm{g} / \mathrm{mL}$ ).

As liver function tests were normal, and serum ammo- 
nia was raised, a diagnosis of valproate induced non-hepatic hyperammonemic encephalopathy was suspected. The valproate was immediately discontinued and replaced by levetiracetam along with L-carnitine supplementation. With these interventions, patient showed rapid recovery over next 4 days and became well oriented, alert and attentive. On repeat estimation, serum ammonia level declined to the normal level $(26 \mu \mathrm{mol} / \mathrm{L})$.

\section{Discussion}

The index case was a child on long-term valproate therapy, presented with clinical features of metabolic encephalopathy, hyperammonaemia and no signs of liver failure. His symptoms were relieved after discontinuation of VPA, with the normalization of serum ammonia level suggesting a clinical diagnosis of VHE. Although, valproate-induced hepatic dysfunction leading to encephalopathy is a known complication, VHE without liver disorder is relatively rare and serious side effect of valproate therapy and only a few case reports are available on occurrence of VHE following chronic use of VPA [3-4]. The typical presentation of VHE include acute onset of impaired consciousness, confusion and lethargy, which may progress to stupor and coma. Focal neurological symptoms and increased seizure frequency may be present [1]. In chronic therapy, onset can be insidious. Although, the incidence of VHE is unknown, mild asymptomatic elevations in serum ammonia have been described in $16 \%-52 \%$ of patients receiving valproate therapy [5].

Other causes of hyperammonemia without hepatic dysfunction include inborn errors of metabolism (e.g. urea cycle enzyme defects, fatty acid oxidation defects), drugs (e.g. 5-FU, asparaginase, salicylate, halothane and enflurane), hematologic diseases like multiple myeloma and acute myeloblastic leukemia, hyperinsulinemia, hyperglycaemia, distal renal tubular acidosis, parenteral nutrition and Reye's syndrome [6]. In our patient, we found urea cycle defects and other metabolic disorders very unlikely and we did not do an extensive metabolic workup in our patient, as there was no previous history of protein intolerance and no reported neonatal, adolescent, or adult deaths in the family. We decided to first withdraw VPA to assess the response as it is known to produce hyperammonemia and our approach was justified by the dramatic clinical improvement after drug withdrawal. We would have investigated the underlying metabolic disorders in case of less dramatic response. Serum valproate level was within normal limits, although there is little correlation between valproate level and clinical effects because of its variable absorption and short half-life [7]. It also correlates poorly with the occurrence of hyperammonemia [8-9].

Several mechanisms have been proposed to explain VPA-induced hyperammonaemic encephalopathy e.g. changes in dietary protein intake, increased production of ammonia in the kidney by VPA and inhibition of mitochondrial carbamoyl phosphate synthetase enzymes in the liver by active metabolites of VPA, which is necessary for ammonia elimination via urea cycle [4, 10]. Additionally, chronic valproate therapy increases the amount of VPA oxidation and production of active metabolites in the liver [11]. Also, chronic VPA therapy enhances urinary excretion of L-carnitine, resulting in depletion of blood carnitine stores leading to reduced capacity of ammonia metabolism [12].

Concomitant use of other AEDs like topiramate, carbamazepine and phenobarbitone may elevate the free biologically active fraction of valproate leading to hyperammonemia [5, 13]. Other risk factors for the development of VHE include young age, multiple neurological disabilities, and poor nutritional intake [14]. High cerebral levels of ammonia leads to an increase in glutamine level in the brain due to increased production and decreased excretion in the astrocytes, which in turn leads to astrocyte swelling and cerebral oedema [15]. Also, increase in activation of gamma-aminobutyric acid (GABA) by ammonia induces somnolence [5]. Treatment for VHE includes supportive care and withholding valproate. Lcarnitine is frequently used to treat VHE [16-17]. Extremely high concentrations of ammonia may warrant dialysis [18].

\section{Conclusion}

VHE is a rare and serious side effect of valproic acid and it may be overlooked when the serum valproate level and liver function tests are within the normal ranges. Thus, we conclude that a high index of suspicion is required for an early recognition and treatment of this potentially life-threatening condition. In a patient taking VPA and presenting with encephalopathy or unexplained increase in seizure frequency, ammonia levels should be checked and valproic acid should be discontinued.

\section{Authorship Contributions}

RA: Patient management, acquisition of data, drafting the article, and literature review; AA: Concept, manuscript review, manuscript editing, revising the article critically for important intellectual content; AA will act as guarantor. Both the authors approved the final manuscript.

\section{Conflict of Interested}

None.

\section{References}

1. Coulter DL, Wu H, Allen RJ. Valproic acid therapy in 
childhood epilepsy. JAMA. 1980;244(8):785-788.

2. Rath A, Naryanan TJ, Chowdhary GV, Murthy JM. Valproate-induced hyperammonemic encephalopathy with normal liver function. Neurol India. 2005;53(2):226228.

3. Cuturic M, Abramson RK. Acute hyperammonemic coma with chronic valproic acid therapy. Ann Pharmacother. 2005;39(12):2119-2123.

4. Stewart JT. A case of hyperammonemic encephalopathy after 11 years of valproate therapy. J Clin Psychopharmacol. 2008;28(3):361-362.

5. Verrotti A, Trotta D, Morgese G, Chiarelli F. Valproateinduced hyperammonemic encephalopathy. Metab Brain Dis. 2002;17(4):367-373.

6. Alqahtani S, Federico P, Myers RP. A case of valproateinduced hyperammonemic encephalopathy: look beyond the liver. CMAJ. 2007;177(6):568-569.

7. Lundberg B, Nergardh A, Boreus LO. Plasma concentrations of valproate during maintenance therapy in epileptic children. J Neurol. 1982;228(2):133-141.

8. DeWolfe JL, Knowlton RC, Beasley MT, Cofield S, Faught E, Limdi NA. Hyperammonemia following intravenous valproate loading. Epilepsy Res. 2009;85(1):65-71.

9. Mehndiratta MM, Mehndiratta P, Phul P, Garg S. Valproate induced non hepatic hyperammonaemic encephalopathy (VNHE)--a study from tertiary care referral university hospital, north India. J Pak Med Assoc. 2008;58(11):627-631.

10. Marini AM, Zaret BS, Beckner RR. Hepatic and renal contributions to valproic acid-induced hyperammonemia. Neurology. 1988;38(3):365-371.

11. Gram L, Bentsen KD. Valproate: an updated review. Acta Neurol Scand. 1985;72(2):129-139.

12. Triggs WJ, Gilmore RL, Millington DS, Cibula J, Bunch TS, Harman E. Valproate-associated carnitine deficiency and malignant cerebral edema in the absence of hepatic failure. Int J Clin Pharmacol Ther. 1997;35(9):353-356.

13. Zaccara G, Paganini M, Campostrini R, Moroni F, Valenza T, Messori A, Bartelli M, et al. Effect of associated antiepileptic treatment on valproate-induced hyperammonemia. Ther Drug Monit. 1985;7(2):185-190.

14. Duarte J, Macias S, Coria F, Fernandez E, Claveria LE. Valproate-induced coma: case report and literature review. Ann Pharmacother. 1993;27(5):582-583.

15. Vossler DG, Wilensky AJ, Cawthon DF, Kraemer DL, Ojemann LM, Caylor LM, Morgan JD. Serum and CSF glutamine levels in valproate-related hyperammonemic encephalopathy. Epilepsia. 2002;43(2):154-159.

16. Lheureux PE, Penaloza A, Zahir S, Gris M. Science review: carnitine in the treatment of valproic acid-induced toxicity - what is the evidence? Crit Care. 2005;9(5):431440.

17. Russell S. Carnitine as an antidote for acute valproate toxicity in children. Curr Opin Pediatr. 2007;19(2):206210.

18. Tsai MF, Chen CY. Valproate-induced hyperammonemic encephalopathy treated by hemodialysis. Ren Fail. 2008;30(8):822-824. 